\title{
Evaluation of Coordination between Cultural Tourism and Urban Economy of Gansu
}

\author{
Rui Lyu ${ }^{1, \mathrm{a}}$, Zhiqiang Liu ${ }^{1, \mathrm{~b}}$ \\ ${ }^{1}$ School of Economics and Management Lanzhou Institute of Technology, Lanzhou, China
}

\begin{abstract}
As Gansu accelerates the building of green eco-industrial system, improving the quality and efficiency of cultural tourism industry has become an important driving force for the transformation of economic quality. To effectively optimize economic structure with tourism resources, a measurement index system of cultural tourism and urban economic development is established, based on which the coordination development model on cultural tourism and urban economy of Gansu is established. The paper can provide theoretical basis for evaluating, tracking and comparing the coordination level between cultural tourism and urban economy of Gansu. It can provide city managers with decision-making basis on developing cultural tourism, protecting cultural heritage and enhancing the comprehensive urban development level.
\end{abstract}

\section{Introduction}

Under the great opportunities provided by Belt and Road Initiative (BRI), cultural tourism has become one of the ten pillar industries to build the eco-industrial system and promote green development of the Gansu. In June 2018, the General Office of Gansu Province Government issued the Special Action Plan for the Development of Cultural Tourism Industry in Gansu Province, which has vigorously promoted the deep integration between culture and tourism and boosted the transformation of cultural tourism industry. Contributing 7\% of the province's GDP in 2018, cultural tourism has become the pillar industry of socio-economic development. In May 2019, the Gansu Provincial Department of Culture and Tourism issued the Action Plan on Improving the Quality and Efficiency of Cultural Tourism Industry in Gansu Province (2019), taking six major actions to transform cultural tourism into connotative growth[1-3]. Gansu enjoys high reputation worldwide for its Silk Road Civilization and Dunhuang Culture and ranks 5th in China for abundance of tourism resources and 6th for tourism growth rate in 2018. But due to various constraints like economic foundation and development level of cultural tourism, the per capita consumption in 2018 was only 682 yuan or $67 \%$ of the national average, which is still at the bottom of the league. Gansu needs to consider how to transform development model and engine and turn its resource advantage into urban economy advantage and competitive edge while developing cultural tourism so as to optimize economic structure and achieve connotative growth of cultural tourism[4-5]. Cultural tourism has become the strategic pillar industry of China's economic development[6-8], under such context, the development of urban and rural tourism industry has a positive influence on urban expansion and the contribution of tourism industry to urban economy is proven in China and beyond[9].

\section{Research Method and Data Sources}

This study aims to explore the internal law of Gansu's urban cultural tourism and compare it with the urban development level. Given the complexity of the index system and the availability of data, cities are selected with the following criteria: tourism revenue should cover over $5 \%$ of the GDP; the city is home to one or more major historical and cultural sites protected at the national level; the city should be China's outstanding tourism city or famous historical and cultural city. The research measures and evaluates the coordination degree between cultural tourism and urban economy of these cities. According to the above criteria, Tianshui, Wuwei, Zhangye and Jiuquan are selected as cases to measure the cultural tourism development level in Gansu. Data comes from Gansu Development Yearbook, Tianshui Economic Yearbook, Wuwei Statistical Yearbook, Zhangye Statistical Yearbook, Jiuquan Statistical Yearbook, China Tourism Statistics Yearbook and China City Statistical Yearbook from 20082018. Besides, some data are accessible on the official websites of the China National Tourism Administration and Gansu Provincial Department of Culture and Tourism. The missing data are estimated with a weighted average of the available data.

aylr0319@163.com blzqzailushanghuang@163.com 


\section{Measurement of Cultural Tourism and Urban Economic Development}

\subsection{The Construction of the Index System and Measurement of Cultural Tourism}

The quantitative evaluation index system of cultural tourism development level is established by referring to related papers, consulting experts in Gansu and beyond and considering the availability of prefectural-level data. A total of 240 questionnaires (60 to each of the four cities) were distributed to the staff of Cultural Tourism Administration Department, the managers of the tourism enterprises in these cities and associate professors or above majoring in tourism management in Gansu and 214 valid questionnaires were collected. The consistency, construct validity and independence of 37 indicators in the questionnaire were tested with SPSS23.0 and then exploratory factor analysis was performed. Three indicators were eliminated through principal component analysis, thus the three-level index system with 5 subsystems, 15 criterion layers and 34 indicators was identified. Entropy method was adopted to assign values objectively and to eliminate the influence of different dimensions and attributes, min-max standardization was adopted so that the development trend of different systems can be reflected. Urban cultural tourism development level of the case cities was measured with TOPSIS method. To obtain the overall ranking, the average value of each indicator was adopted and the ranking is shown in Table 1.

Table1. the Coordination Degree between Cultural Tourism and Urban economy

\begin{tabular}{|c|c|c|c|c|}
\hline City & D+ & D- & Si & Ranking \\
\hline Tianshui & 0.1028 & 0.0361 & 0.2599 & 3 \\
\hline Wuwei & 0.1335 & 0.0282 & 0.1744 & 4 \\
\hline Zhangye & 0.0705 & 0.1034 & 0.5946 & 2 \\
\hline Jiuquan & 0.0676 & 0.1085 & 0.6161 & 1 \\
\hline
\end{tabular}

According to the data in Table 1, the analysis of the development level of cultural tourism in case cities is as follows:

With one district, two cities and four counties, Jiuquan enjoys rich tourism cultural resources. It is home to one 5A-rated tourist attraction, two 4A-rated tourist attractions and a 3A-rated tourist attractions. As a world cultural heritage site, Mogao Caves have already become a namecard for Gansu tourism since last century. Natural scenery and historical and cultural resources are the foundations for quality cultural tourism development of Jiuquan. In recent years, through the development of major and special scenic sites and special tourist towns, regional tourism has been further integrated and smart tourism has been improved. Western outdoor sports in China (Jiuquan) has taken initial shape, thus cultural tourism industry in Jiuquan shows a diversified and fast development trend.

In recent years, on the basis of the historical and cultural city, Zhangye has embarked on the path of deep integration between tourism and culture, sport and medical care. To develop the major tourism landscape and promote the integration of scenic spot resources, Zhangye Cultural
Tourism Integrated Development Entrepreneurship Demonstration Base, Ganzhou District Folkway Culture Industrial Park, Gaotai Recreation and Tourism Entrepreneurship Base and Zhangye Outdoor Sports and Sport Industry Creative Park have been constructed. The development of major scenic sites has effectively integrated different tourism resources and enhanced the reputation and influence of Zhangye- China's Outstanding Tourism City.

Tianshui ranks third among the four cities on cultural tourism development and its $\mathrm{Si}$ value decreases significantly compared with the top two cities. The main image of Tianshui on cultural tourism is "hometown of Xihuang" and the city is endowed with rich natural and cultural heritage. In recent years, cities along the Hexi Corridor have jointly built the northwestern regional tourist distribution center, which has rapidly increased the attention to places like Jiuquan, Zhangye and Wuwei, thus Tianshui has fewer location advantages. But it enjoys fast development in cultural undertakings and has won the award of "China's Most Attractive Festival City" and Fuxi Culture and Tourism Festival has won the title of "the most Influential Festival". The strengthened cultural soft power of Tianshui has laid a foundation for further developing cultural tourism with unique features.

The Si value of Wuwei has little difference with that of Tianshui. But Wuwei has relatively few tourism resources and weak industry foundation, thus the market development is limited. To boost the development of cultural tourism, Wuwei has proposed competitive industry, happiness Industry and strategic pillar industry, integrated its special tourism resources like history, culture, natural scenary and industry with unique features and optimized industrial spatial distribution, thus new tourism forms are cultivated.

\subsection{The Construction of Index System and Measurement of Urban Economic Development}

Since researches on measuring urban economic development level have become relatively mature, this paper selects the most frequently used system with 3 categories and 8 indexes after referring to relevant studies. Similarly, entropy method was adopted to assign values objectively. The measurement results of urban economic development level are shown in Figure 1, from which we may find that Jiuquan, Zhangye, Tianshui and Wuwei have all improved greatly. On the whole, Tianshui ranks first, followed by Zhangye, Jiuquan and Wuwei, respectively. From the perspective of socio-economic development level, the ranking is not so consistent with that of cultural tourism. 


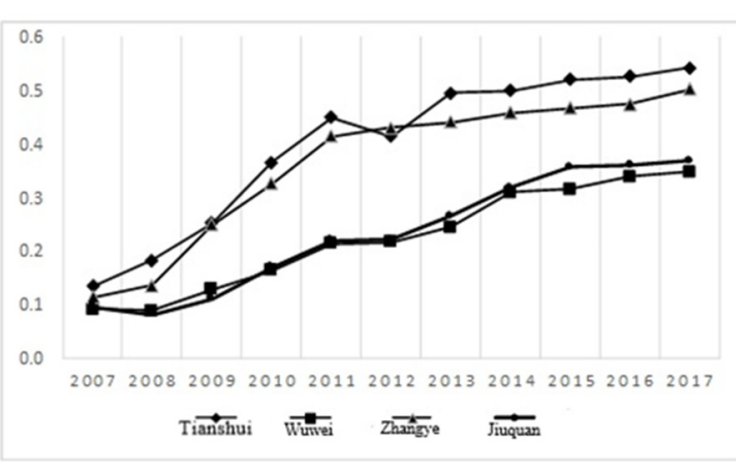

Fig1. Urban Economic Development level of Case Cities (2007-2017)

\section{Measurement of the Coordination Level}

\subsection{Construction of the Coordination Development Model}

Cultural tourism and urban economic development are interactive, but how do they interact with each other in Gansu? Are they coordinated? This paper attempts to construct a coupling coordination model with reference to related studies to measure the coordination degree between cultural tourism and urban economy in Gansu. Coupling coordinating degree can reflect the strength of interaction of the two systems. The greater the coupling coordination degree is, the more coordinated the interaction; the smaller the degree is, the more one-sided the development. For example, urban development relies on cultural tourism but at the same time constraints the upgrading of cultural tourism or urban development is rapid but it doesn't effectively use the resources of cultural tourism. The specific coupling coordination model is as follows:

$$
\begin{gathered}
\mathrm{C}=\frac{F_{1} \times F_{2}}{\left[F_{1}+\mathrm{F}_{2}\right]^{2}} \\
\mathrm{D}=\sqrt{C \times T} \\
\mathrm{~T}=\alpha \mathrm{F}_{1}+\beta \mathrm{F}_{2}
\end{gathered}
$$

Where D represents the coupling coordination degree between cultural tourism and urban economy; $\mathrm{C}$ represents the coupling degree; $\mathrm{T}$ represents overall evaluation index of the coordination between the two systems, indicating their comprehensive development level; $F_{1}$ and $F_{2}$ represent the comprehensive evaluation indexes of the two systems respectively; $\alpha, \beta$ are the weight coefficients. $T \in(0,1), D \in(0,1)$, and after referring to relevant studies, the weights of $\alpha$ and $\beta$ are set as 0.4 and 0.6 respectively.

Table2. the Coordination Degree between Cultural Tourism and Urban economy

\begin{tabular}{|c|c|c|}
\hline Grade & Coordination degree & Grade feature \\
\hline I & $(0.85,1]$ & Quality coordination \\
\hline II & $(0.7,0.85]$ & Good coordination \\
\hline
\end{tabular}

\begin{tabular}{|c|c|c|}
\hline Grade & Coordination degree & Grade feature \\
\hline I & $(0.85,1]$ & Quality coordination \\
\hline III & $(0.55,0.7]$ & Basic coordination \\
\hline IV & $(0.4,0.55]$ & Bare coordination \\
\hline V & $(0.25,0.4]$ & Mild imbalance \\
\hline VI & $(0.1,0.25]$ & Moderate imbalance \\
\hline VII & {$[0,0.1]$} & Extreme imbalance \\
\hline
\end{tabular}

\subsection{Coupling Coordination Degree}

Development level of cultural tourism subsystem $F_{1}$ and that of urban economy subsystem $F_{2}$ have been obtained by standardizing relevant data, assigning value with entropy method and counting the weights. Coupling coordination degree between cultural tourism and urban economy in Gansu are calculated according to related formula. The results can be found in Table 3 .

Table3. the Coordination Degree between Cultural Tourism and Urban economy

\begin{tabular}{|c|c|c|c|c|}
\hline $\begin{array}{c}\text { City } \\
\text { Year }\end{array}$ & Tianshui & Tianshui & Tianshui & Tianshui \\
\hline 2007 & 0.2741 & 0.2155 & 0.2631 & 0.1917 \\
\hline 2008 & 0.3143 & 0.2526 & 0.3124 & 0.2644 \\
\hline 2009 & 0.3109 & 0.2962 & 0.3237 & 0.2083 \\
\hline 2010 & 0.3872 & 0.3226 & 0.3732 & 0.3155 \\
\hline 2011 & 0.4158 & 0.3731 & 0.4275 & 0.3721 \\
\hline City & Tianshui & Tianshui & Tianshui & Tianshui \\
\hline Year & & & & \\
\hline 2012 & 0.4373 & 0.4123 & 0.4984 & 0.4087 \\
\hline 2013 & 0.4533 & 0.4572 & 0.5136 & 0.4797 \\
\hline 2014 & 0.4734 & 0.4957 & 0.5318 & 0.487 \\
\hline 2015 & 0.4982 & 0.5483 & 0.6047 & 0.5009 \\
\hline 2016 & 0.5031 & 0.5957 & 0.6219 & 0.5288 \\
\hline
\end{tabular}

According to the data in Table 3, the case cities grew from imbalance to coordination on cultural tourism and urban economy from 2007 to 2017. During this period, Tianshui developed from mild imbalance to bare coordination; Wuwei changed from moderate imbalance to basic coordination; Zhangye grew from mild imbalance to basic coordination; Jiuquan developed from moderate imbalance to bare coordination. From the table we can know: in recently 10 years, the coordination level between cultural tourism and urban economy in Gansu is increasing on the whole and has generally got rid of imbalance; the coupling coordination degree of the two systems have grown from imbalance to coordination, but even those cities with better development are still at the basic coordination stage, thus the overall coordination needs to be improved. After 2010, the coordination of the two systems entered a fast development phase, but comparing related research, we find that the coupling coordination degree of the two systems of eastern cities have already been over 0.85 ten years ago. There is an urgent need for Gansu to improve the quality and performance of its cultural tourism and make it the pillar industry for green development. 


\section{CONCLUSION}

For Tianshui, its $\mathrm{F}_{1}>\mathrm{F} 2$ from 2007 to 2013 , indicating that the urban economy lags behind cultural tourism. As for urban development, Tianshui ranks first among the four cities. Through the advocation of Fuxi culture and festival activities, better cultural identification is achieved, making the cultural tourism enjoy a fast development. In April 2012, Publicity Department of Lanzhou Municipal Committee and Tourism Bureau established the slogan "Travel in Northwestern China, Start from Lanzhou" for the promotion of the city's tourism. The slogan has an influence on the tourism industry of Tianshui, which lies in the southeast of Gansu. On the whole, the coupling coordination degree of the two systems is rising, but it is only barely coordinated and needs to be improved.

For Wuwei, its unban economic development lags behind that of cultural tourism in 2007-2009, 2015 and 2016, but in the rest years, cultural tourism lags behind urban economy. Besides, the overall performance of the two systems in Wuwei is the weakest among the four cities, but its coupling coordination degree is rising year by year. It has grown from moderate imbalance to basic coordination, a better coordination level in the four cities. Wuwei is developing quality cultural tourism to pillar industry, which has boosted regional economic development.

For Zhangye, its cultural tourism development lags behind that of urban economy in 2007 and 2008. In 2009, measures on integration between culture and tourism were introduced. As the Oasis Beyond the Great Wall, Zhangye has abundant cultural tourism resources and has proposed to follow the path of deep coordination between tourism and culture, sport and medical care, thus the cultural tourism has achieved fast development.

The unique cultural tourism resource explains why its urban economy lags behind for many years. The coupling system is developing from moderate imbalance to bare coordination slowly. During the 13th Five-Year Plan period (2016-20), Jiuquan proposed to develop tertiary industry and become a better city, seize the strategic opportunities provided by BRI and Chinese Civilization Inheritance and Innovation Zone and promote the integration of cultural tourism and sport industry so as to transform from a cultural tourism city of quantity to a cultural tourism city of quality. In conclusion, to further improve the coordination of the two systems is a common task for cities in Gansu when developing cultural tourism and regional economy.

\section{Acknowledgment}

This research is supported by the project of "2019 Gansu Provincial Higher Education Innovation Capacity Enhancement Project (2019A-147) ", and "The 'Qizhi' Talent Cultivation Project of Lanzhou Institute of Technology (2018QZ-12) ".

\section{References}

1. ZHANG Jing, LI Qiang, LI Shiyu, "Study on Residents' Perceptions of Tourism Impact at the World Heritage Site: Villagers of Zhaoling village and Changling Village in Shisanling Town, Changping District, Beijing as the Case," Urban Development Studies, Vol.26, pp. 33-36, No.05 2019.

2. LIU Jiayi, ZHAO Lei, SHEN Weili, "Will Tourism Development Drive Urban Spatial Expansion?" Tourism Tribune, Vol. 33, pp. 41-50, No. 10, 2018.

3. CHEN Mei, ZHAO Weitao, "A Study on the Relationship between Eco-efficiency and Tourism under the New-type Urbanization: A Case Study of Urban Clusters in the Middle Reaches of the Yangtze River," Tourism Tribune, Vol. 33, pp. 89-97, No.3,2018.

4. LIU Minkun, JIANG Liling, "A Review of Research on the Contribution of Tourism to Economic Growth," Tourism Tribune, Vol. 32, pp. 33-42, No.4,2017.

5. LIU Minkun, JIANG Liling, "A Review of Research on the Contribution of Tourism to Economic Growth," Tourism Tribune, Vol. 32, pp. 33-42, No.4,2017.

6. HE Jinliao,ZENG Gang, "Dynamic Mechanism of Creative Industry Agglomeration under the Driving of Urban Amenities: A Case Study of Brand Design Industry in Nanjing," Economic Geography, Vol. 39, pp. 134-142,161 No.3, March 2019.

7. MA Renfeng, WANG Tengfei, ZHANG Wenzhong, "Gentrification mechanism of the old industrial districts in Ningbo from the perspective of creative regeneration," ACTA Geographica Sinica, Vol.28, pp. 780-796, No.4, April 2019.

8. WANG Mengting et al, "Research on the development path of cultural tourism industrial park from the perspective of the integration of culture and tourism-Taking the Songshan cultural industry park as an example," Territory \& Natural Rsources Study, pp. 65-67, No.6, 2018.

9. LIU Yang, LI shumin, "Urban Management and the Coordinated Development of Cultural Tourism," Journal of Qiqihar University( Phi \& Soc Sci), pp. 49-51,66, No.11, November 2015 